\title{
MANIFOLDS WITH THE FIXED POINT PROPERTY. I
}

\author{
BY S. Y. HUSSEINI ${ }^{1}$
}

Communicated by Mary Ellen Rudin, November 6, 1973

1. Introduction. Suppose that $f: M \rightarrow M$ is a map of the simply connected closed (smooth or PL) manifold $M$ which preserves a given geometric structure. We shall consider the question of when $f$ has a fixed point. (The geometric structure is described by an element $\xi$ in $K_{R}(M)$, the Grothendieck group of real vector bundles over $M$. If $\operatorname{deg} f=1$, then for $f$ to preserve $\xi$ means just that $f^{*} \xi=\xi$, and the appropriate notion when $\operatorname{deg} f \neq 1$ is given below in $\S 2$. Such maps are said to be $(\xi, \lambda)$-maps with $\lambda$ an integer.) Since $M$ is simply connected, one need only compute the Lefschetz number $\mathscr{L}(f)$ of $f$. Thus there are three natural stages to the solution: the determination of the induced homomorphism $f^{*}: H^{*}(M ; Z) \rightarrow$ $H^{*}(M ; Z)$ first below the middle dimension, then in the middle dimension (when $\operatorname{dim} M$ is even), and finally the determination of how the two are related to each other and how they determine the behaviour above the middle dimension.

As a first step in this direction, we consider here the case of $(2 m-1)$ connected $M$ of dimension $4 m$ whose intersection pairing is definite (said to be of class $\mathscr{M}_{4 m}$ ). It is shown that if $\xi$ is asymmetric enough in a suitable sense (described below in $\S 2$ ), then any $(\xi, \lambda)$-map $f: M \rightarrow M$ has a fixed point. In particular it follows that if the tangent bundle $\tau(M)$ of $M$ is asymmetric enough, then a $(\tau M, 1)$-map $f: M \rightarrow M$ has a fixed point. Therefore every homeomorphism of such a manifold $M$ has a fixed point. It is also shown that the product of $(\xi, \lambda)$-maps with $\xi$ being asymmetric also has a fixed point.

[Note. At this point I would like to thank Ed Fadell for the suggestions and stimulation offered in many good conversations on this topic.]

2. Statement of results. Suppose that $M$ is a smooth (or PL) simply connected closed manifold of dimension $4 m$. A map $f: M \rightarrow M$ is said to be a $(\xi, \lambda)-m a p$, where $\lambda$ is an integer, if and only if $f^{*} \xi=\lambda \xi+p^{*} \eta$ where

AMS (MOS) subject classifications (1970). Primary 55C20, 57D99; Secondary 54H25, $15 \mathrm{~A} 63$.

${ }^{1}$ While working on this paper the author has been partially supported by the National Science Foundation GP-29538-A4.

Copyright @ American Mathematical Society 1974 
$p: M \rightarrow S^{4 m}$ is a map of degree 1 and $\eta \in K_{R}\left(S^{4 m}\right)$. (Note that a diffeomorphism $f: M \rightarrow M$ is a $(\tau M, 1)$-map, $\tau(M)$ being the tangent bundle of M.)

Assume now that $M$ is $(2 m-1)$-connected, and suppose that the intersection pairing

$$
\varphi: H^{2 m}(M ; Z) \times H^{2 m}(H ; Z) \rightarrow Z
$$

is positive definite. The class of such manifolds will be denoted by $\mathscr{M}_{4 m}$. (See [1] for their classification.) Let $c_{m}=c_{m}\left(\xi^{c}\right) \in H^{2 m}(M ; Z)$ be the Chern class of $\xi^{c}=\xi \otimes C$, the complexification of $\xi$, and assume that $c_{m}\left(\xi^{c}\right) \neq 0$. One can easily show that this implies that $\operatorname{deg} f=\lambda^{2}$ and the Lefschetz number $\mathscr{L}(f)$ is given by $\mathscr{L}(f)=1+s \lambda+\lambda^{2}$, with $s$ rational and $|s| \leqq \sigma$, $\sigma$ being the signature of $\varphi$. Hence $\mathscr{L}(f) \neq 0$ for $|\lambda|>\sigma$. On the other hand, the behaviour of $\mathscr{L}(f)$ for $|\lambda| \leqq \sigma$ is quite different, and thus $\sigma$ is, in a sense, a critical threshold.

To describe the case $|\lambda| \leqq \sigma$, one shows first that there is a basis $\mathscr{S}=$ $\left\{x_{1}, \cdots, x_{\sigma}\right\}$ for $H_{2 m}(M ; \bar{Z})$ with the property that $\left\langle x_{i}, c_{m}\right\rangle=\beta^{s_{i}}$ where $\beta=\min \left\langle x, c_{m}\right\rangle$ and $s_{i}$ are integers such that $s_{1}=1, s_{j}-s_{i}>0$ for all $j>i$, and $c_{m}$ the mth Chern class of $\xi$.

The basis $\mathscr{S}=\left\{x_{1}, \cdots, x_{\sigma}\right\}$ defines a critical region for $\xi$. If $x, y \in$ $H_{2 m}(M ; Z)$ and $x y$ denotes their intersection number, then the critical region is the set

$$
B_{\mathscr{S}}=\left\{x \in H_{2 m}(M ; Z) \mid x^{2} \leqq \sigma^{2} \mu_{\mathscr{S}}\right\}
$$

where $\mu_{\mathscr{S}}=\max _{i} x_{i}^{2}$. Now let $\beta_{\mathscr{S}}$ be the smallest integer such that $\left|a_{i}\right|<$ $\beta_{\mathscr{S}}-\sigma$ for all $i$, where $\sum_{i} a_{i} x_{i} \in B_{\mathscr{S}}$. $\xi$ will be said to be sufficiently asymmetric if, and only if, $\beta \geqq \beta_{\mathscr{S}}$.

THEOREM 2.1. Suppose that $\xi$ is sufficiently asymmetric. Then any $(\xi, \lambda)$-map $f: M \rightarrow M$ has a fixed point, where $M \in \mathscr{M}_{4 m}$ and $m>4$.

The following is an immediate consequence.

THEOREM 2.2. Suppose that $M \in \mathscr{M}_{4 m}$ with $m$ even and $>4$, and assume that $\tau(M)$, the tangent bundle of $M$, is sufficiently asymmetric. Then any $(\tau M, 1)$-map $f: M \rightarrow M$ has a fixed point. In particular, any homeomorphism of $M$ has a fixed point.

The next theorem describes the behaviour of the products of $(\xi, \lambda)$ maps.

THEOREM 2.3. Suppose that $M^{\prime}$ and $M^{\prime \prime}$ are two manifolds in $\mathscr{M}_{4 m^{\prime}}$ and $\mathscr{M}_{4 m^{\prime \prime}}$ with $m^{\prime}, m^{\prime \prime}>4$. Let $\xi^{\prime} \in K_{R}\left(M^{\prime}\right)$ and $\xi^{\prime \prime} \in K_{R}\left(M^{\prime \prime}\right)$ be sufficiently asymmetric, and put $\xi=\xi^{\prime} \otimes \xi^{\prime \prime}$ where $\otimes$ is the tensor product. Then any $(\xi, \lambda)$-map $f: M^{\prime} \times M^{\prime \prime} \rightarrow M^{\prime} \times M^{\prime \prime}$ has a fixed point. 
3. Construction of $(\xi, \lambda)$-maps. In view of the preceding, it is important to know whether there is a $(\xi, \lambda)$-map $f: M \rightarrow M$. A map such as $f$ has degree $\lambda^{2}$, and therefore the question becomes whether there is a map $f: M \rightarrow M$ of a given degree and whether a map of a given degree preserves a given $\xi \in K_{R}(M)$. Let therefore $\alpha: H_{2 m}(M ; Z) \rightarrow \pi_{2 m-1} S O$ be the map which associates to $x$ the characteristic class of the induced bundle $g^{*} \tau(M), g$ being an imbedding $S^{2 m} \rightarrow M$ realizing $x$.

THEOREM 3.1. Suppose that $\gamma: H^{2 m}(M ; Z) \rightarrow H^{2 m}(M ; Z)$ is a monomorphism such that $\varphi(\gamma x, \gamma y)=\lambda^{2} \varphi(x, y)$ for all $x, y \in H^{2 m}(M ; Z)$, where $\lambda$ is a given integer and $\varphi$ is the intersection pairing in M. Assume also that $\gamma(\alpha)=\lambda \alpha$. Then there is a map $f: M \rightarrow M$ such that $\gamma$ is the induced homomorphism on cohomology, provided that $J(\lambda(\lambda-1) \alpha(x))=0$ for all $x \in$ $H_{2 m}(M ; Z)$, with $J$ being the J-homomorphism (cf. [2, Lemma 10] and [1, Theorem 5]).

Whether or not a map $f: M \rightarrow M$ of a given degree preserves a given $\xi \in K_{R}(M)$ is decided by considering the characteristic classes of $\xi$ and $f^{*} \xi$.

Thus the question of finding a $(\xi, \lambda)$-map $f: M \rightarrow M$ amounts to finding a homomorphism $\gamma: H^{2 m}(M ; Z) \rightarrow H^{2 m}(M ; Z)$ which preserves the intersection pairing $\varphi$, the stable tangential structure $\alpha$, and the Chern class of $\xi$. If $M$ is almost parallelizable, then $\alpha$ is trivial, $\tau(M)$ has a large measure of symmetry, and the existence of $(\xi, \lambda)$-maps depends only on $\xi$ and how large the group of automorphisms of $\varphi$ is. In particular, if $\lambda=1$ and $\xi=$ $\tau(M)$, it follows that every quadratic automorphism $\gamma: H^{2 m}(M ; Z) \rightarrow$ $H^{2 m}(M ; Z)$ is induced by a corresponding homeomorphism $f: M \rightarrow M$.

\section{REFERENCES}

1. C. T. C. Wall, Classification of (n-1)-connected $2 n$-manifolds, Ann of Math. (2) 75 (1962), 163-189. MR 26 \#3071.

2. - Classification problems in differential topology. II. Diffeomorphisms of handlebodies, Topology 2 (1963), 263-272. MR 27 \#6278.

Department of Mathematics, University of Wisconsin, Madison, Wisconsin 53706 